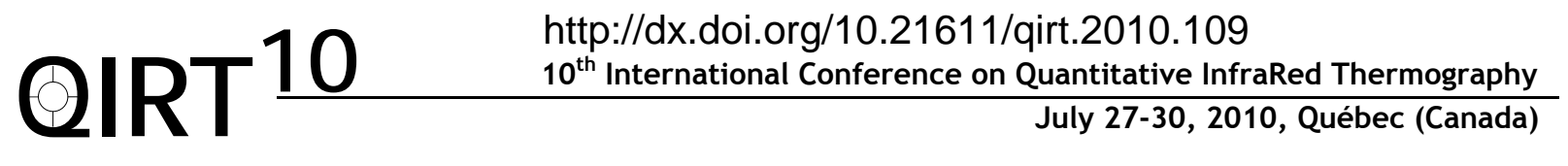

\title{
Transition detection on an Ultra-Streamlined vehicle using IR thermography
}

\author{
by Ph. Planquart*, A. Claesen, J.M. Buchlin**
}

EA Dept., von Karman Institute for Fluid Dynamics, Rhode-Saint-Genèse, Belgium, *planquart@vki.ac.be, **buchlin@vki.ac.be

\begin{abstract}
The World Solar Challenge is a solar-powered car race, which cover $3021 \mathrm{~km}$ from Darwin to Adelaide. Most of the vehicles participating at this race can be defined as Ultra-Streamlined body; the drag being mainly produced by skin friction. In designing such vehicle, it is important to localize the area where transition from laminar to turbulent flow will occur. The location of transition will affect the overall drag coefficient of the vehicle. Moreover its determination by CFD simulation is a challenging task. This paper presents an experimental study based on momentum-heat transfer analogy, which has been carried out in the VKI-L1A subsonic wind tunnel. The infrared thermography is applied on a scaled model of the Umicore solar car, which was designed for the World Solar Challenge.
\end{abstract}

\section{Introduction}

The drag of a ground vehicle consists mainly of skin friction and pressure drag. For Ultra-streamlined vehicle, the drag is mainly produced by skin friction, separation being avoided all the way to the trailing part of the vehicle. Most of the vehicles participating at the World Solar Challenge (Figure 1) can be defined as Ultra-Streamlined body. In designing such vehicle, it is important to localize the area where transition from laminar to turbulent flow occurs. This transition position will affect the overall drag coefficient of the vehicle and is crucial for tuning CFD codes used in the design of the new vehicles.

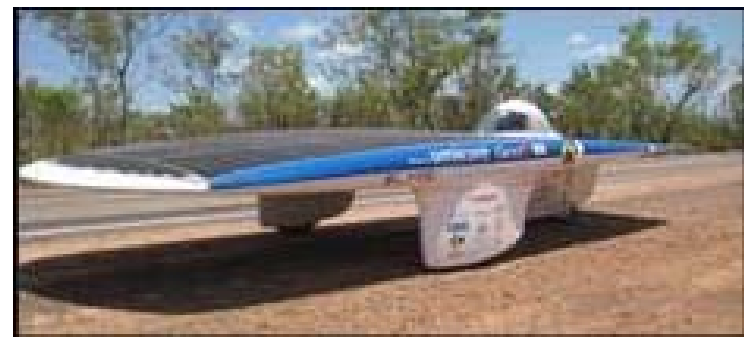

Figure 1 - Ultra-streamlined body

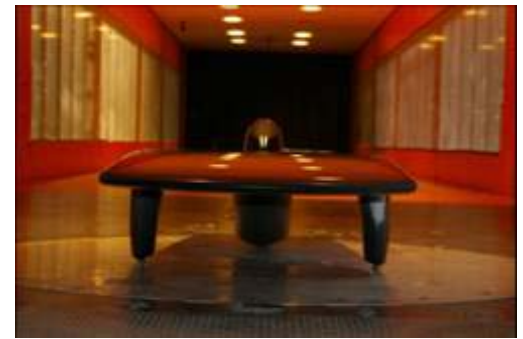

Figure $2-1 / 3$ scaled model in the wind tunnel

In this paper, we present experimental results obtained in the VKI-L1A subsonic wind tunnel using the FLIR ThermaCAM SC 3000 camera on a scaled model of the Umicore Solar Car, shown in Figure 2, which was designed by students of Groep T (Leuven Engineering College, Belgium) for the World Solar Challenge race.

The transition is detected using an unsteady heat transfer approach [1], the model being cooled down before starting the wind tunnel. The time variation of the surface temperature is recorded with the infra-red camera. Using in-house developed post-processing tools, we are able to determine the local heat transfer coefficient and determine the position where the heat transfer increases due to turbulence.

The post-processing is based on the relation for the lumped capacitance model and for the semi-infinite model [2], for which the time dependent surface temperature is a function of the heat transfer coefficient, the material conductivity and the thermal diffusivity of the model, the time, the initial temperature of the model and the air temperature. More details on the post-processing are given in chapter $n^{\circ} 3$.

\section{Methodology and description of the experiments}

Three different sets of experiments have been performed:

1- First, to validate the methodology and the post-processing tools, the model was placed outside the wind tunnel and heat transfer coefficient values obtained are compared to reference value for natural convection. To cool down the 
model, ice was first placed in a plastic bag on top of the model. A surface temperature of $4{ }^{\circ} \mathrm{C}$ is obtained at the beginning of the test, and using the reference value of heat transfer in natural convection, we were able to extract the thermal material properties. Latter, the model was placed in a box filled with dry-ice to decrease even more the initial model temperature. An initial temperature of $-7^{\circ} \mathrm{C}$ has been recorded.

2- Afterwards, a series of test was performed in the wind tunnel with the camera placed on the side of the wind tunnel and inclined, as represented in Figure 3. The model was still cool down by a bag filled with ice cubes. A typical surface temperature map obtained for a wind tunnel speed of $50 \mathrm{~m} / \mathrm{s}$ is shown in Figure 4 . In this figure, the flow is going from right to left. The noise of car is located on the right of the picture. In the experiment, a string was placed on $1 / 3$ of the width of the car (upper part of the figure) to force a turbulent flow. The impact of this string on the temperature map (heat transfer coefficient) is clearly identified. Heat transfer coefficients of $30 \mathrm{~W} / \mathrm{m}^{2} \mathrm{~K}$ and $50 \mathrm{~W} / \mathrm{m}^{2} \mathrm{~K}$ are respectively obtained for the laminar and the turbulent region.

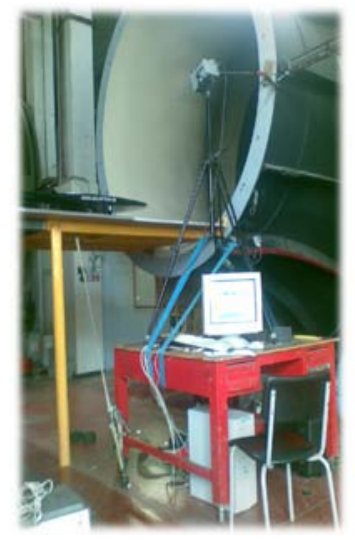

Figure 3 - Initial test set-up

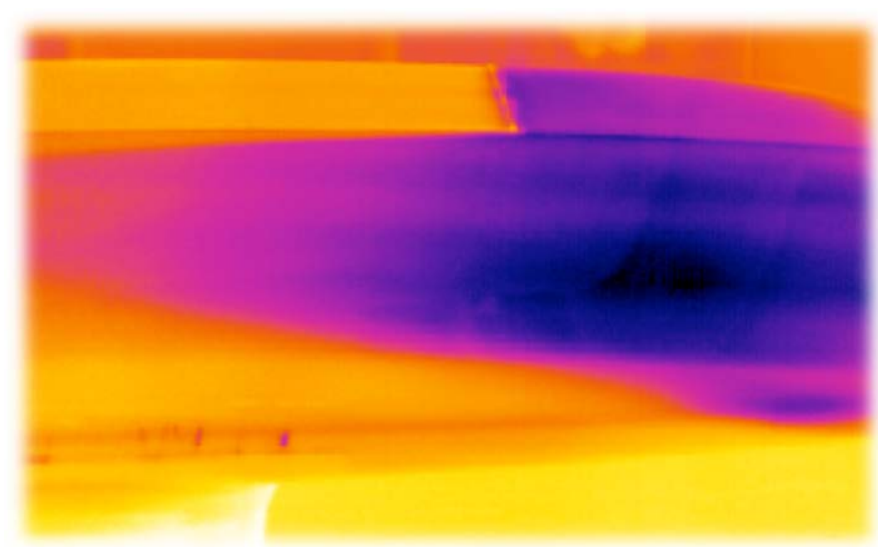

Figure 4 - Instantaneous temperature $-U=50 \mathrm{~m} / \mathrm{s}$

3- Finally, the camera is placed above the model (Figure 5) and the model is cool down using dry ice. A special box was therefore build to cover the whole model and this box was filled with dry ice to get a uniform initial surface temperature at around $-7^{\circ} \mathrm{C}$. Two different scaled models of a solar-powered car haven been studied.

Figure 6 shows the surface temperature distribution on the first model for a wind speed of $50 \mathrm{~m} / \mathrm{s}, 120$ seconds after the start of the wind tunnel. The flow is going from left to right. The blue part on the upper and lower part of the image corresponds to the wind tunnel floor temperature. The blue part on the left of the picture (cold part) represents the region where the flow remains laminar. Transition begins in the green area and in the red part, the flow is fully turbulent. Vortices on the lateral part of the car are responsible for the turbulent region observed on both sides of the car up to the cockpit. The transition is therefore induced by the 3D flow field produced by the bottom part of the model. The cockpit (red part on the right of the picture) influences the flow structure and creates a region of high heat transfer coefficient. It is worth mentioning that the flow pattern observed using IR thermography is similar to the one observed using oil flow visualization around the cockpit.

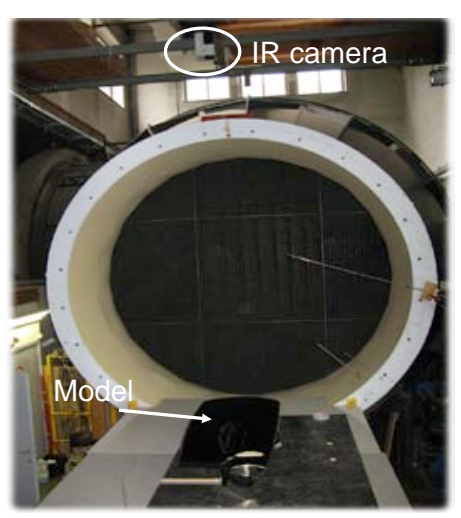

Figure 5 - Final test set-up

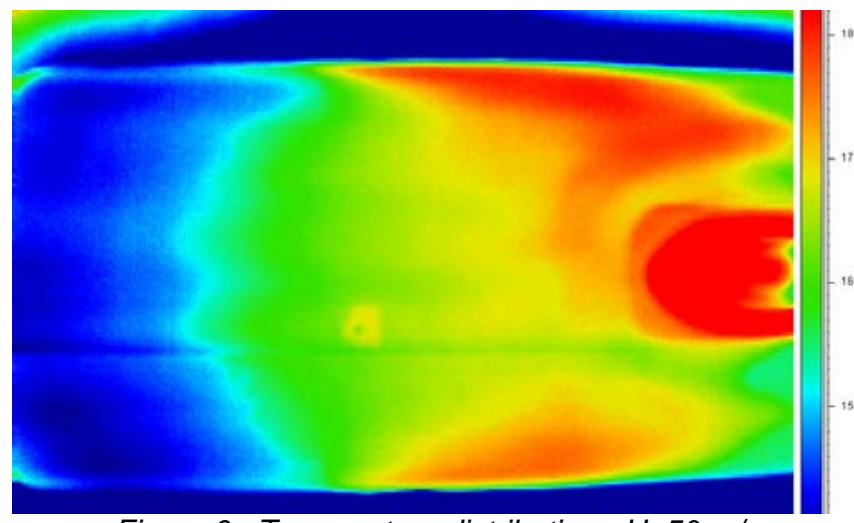

Figure 6 - Temperature distribution - $U=50 \mathrm{~m} / \mathrm{s}$ 


\section{Quantitative results and Post processing}

The post-processing is based on the relation for the lumped capacitance model and semi-infinite model [2], for which the time dependent surface temperature is a function of the heat transfer coefficient, the material properties of the model, the time, the initial temperature of the model and the air or fluid flow temperature.

The Biot number based on the model thickness will allow us to choose between the lumped capacitance model and the semi-infinite model for the post-processing of the results.

Equation $n^{\circ} 1$ represents the equation of the lumped capacitance model, which can be applied when the temperature gradients inside the body have all decayed and the body temperature can be approximated by a single value $\mathrm{T}(\mathrm{t})$.

$$
\frac{T-T_{\infty}}{T_{1}-T_{\infty}}=\exp \left[\frac{-h A}{\rho c V}\left(t-t_{c}\right)\right]=\exp \left[-X\left(t-t_{c}\right]\right.
$$

Equation $n^{\circ} 2$ represents the equation of the semi-infinite model, called also the early regime model, for which the skin temperature is different than the temperature inside the material. Equation $\mathrm{n}^{\circ} 2$ provides the skin temperature $\mathrm{T}(0, \mathrm{t})$ as function of time.

$$
\frac{T(0, t)-T_{\infty}}{T_{1}-T_{\infty}}=-\exp \left[\frac{-h^{2} \alpha t}{k^{2}}\right] \operatorname{ercf}\left(\frac{h}{k} \sqrt{\alpha t}\right)
$$

For both equations, the knowledge of the surface temperature in function of time allows us to solve the timedependent equations for every time step and compute the local heat transfer coefficient, which should be independent of time, as the flow field is stationary once the wind tunnel has reached steady-state conditions.

The following results have been obtained using the second scaled model built for the World Solar Challenge of 2009. Figure 1 shows the temperature map recorded 10 seconds after the wind tunnel has reached a stationary wind speed of $40 \mathrm{~m} / \mathrm{s}$. The flow is going from the bottom to the top of the image. The shape of the car can clearly be identified. A string is located on the right part of the model to force the transition from laminar to turbulent. Temperature evolutions as function of time in the laminar region and in the turbulent region (both sides of the string) are shown respectively in Figure 8 and in Figure 9.

The theoretical model used in Figure 8 and in Figure 9 is the lumped model (Equation $n^{\circ} 1$ ), for which the term in the exponential has been adapted to obtain the best agreement with the experimental points. The model follows quite well the time-dependent temperature evolution.

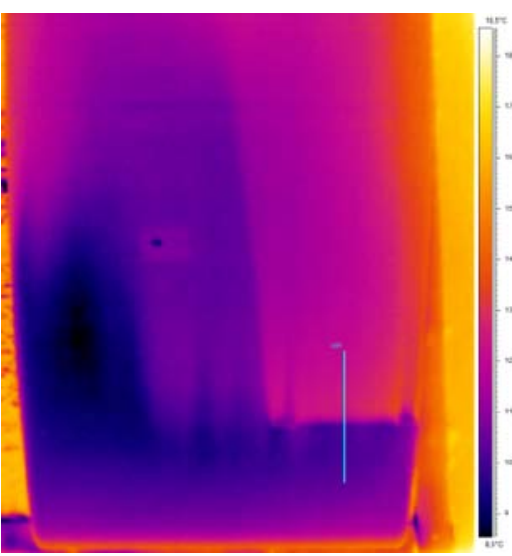

Figure 7 - Temperature map $U=40 \mathrm{~m} / \mathrm{s}$

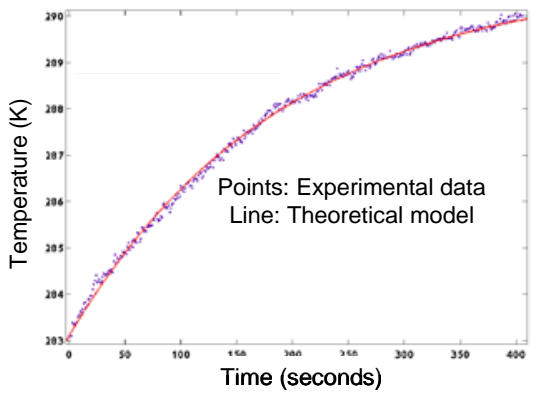

Figure 8 - Temperature evolution laminar region

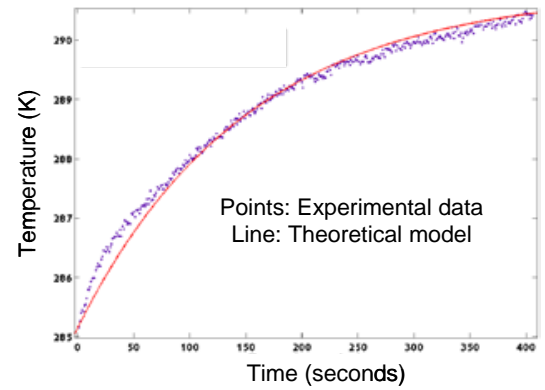

Figure 9 - Temperature evolution turbulent region 
The ratio between the heat transfer coefficient in the laminar region and in the turbulent region can be deduced from the ratio of the $X$ term in the exponential part of the lumped model (Equation $n^{\circ} 2$ ): The $X$ term being different in the laminar and turbulent region (Equation $n^{\circ} 3$ and equation $n^{\circ} 4$ ).

$$
\begin{aligned}
& X_{\text {lam }}=\left[\frac{h_{\text {lam }} A}{\rho c V}\right] \\
& X_{\text {turb }}=\left[\frac{h_{\text {turb }} A}{\rho c V}\right]
\end{aligned}
$$

A ratio of 1.31 is obtained for the ratio of $\frac{X_{\text {turb }}}{X_{\text {lam }}}$ or for the ratio of the turbulent to the laminar heat transfer coefficient.

A second series of tests has been performed with the same model but by changing slightly the angle of attack of the model. During previous tests on the VKI vehicle aerodynamic balance, we have put in evidence that a slight change in the angle of attack influences significantly the total drag of the vehicle. The results shown in Figure 10 and in Figure 11 illustrate that the transition from laminar to turbulent has moved forward, compared to the previous experiments. The circle in Figure 10 and Figure 11 defines the position at which the transition has been detected on the green line.

The transition moves forward with an increase of the wind speed. At a wind speed of $40 \mathrm{~m} / \mathrm{s}$, the laminar region is quite small and the length of the laminar zone is not uniform along the width of the model.

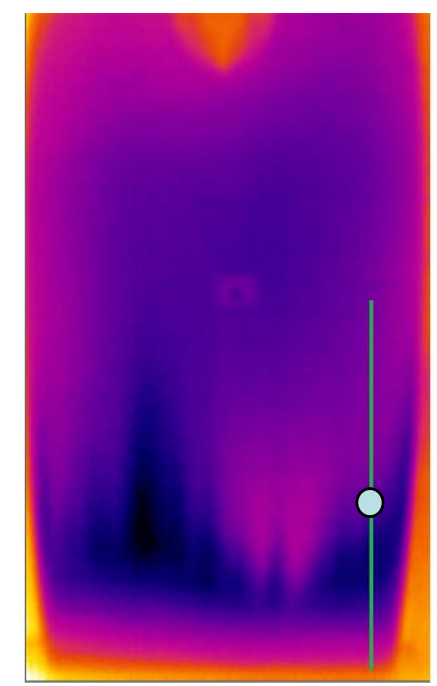

Figure 10 - Test $n^{\circ} 2-\mathrm{U}=32.5 \mathrm{~m} / \mathrm{s}$

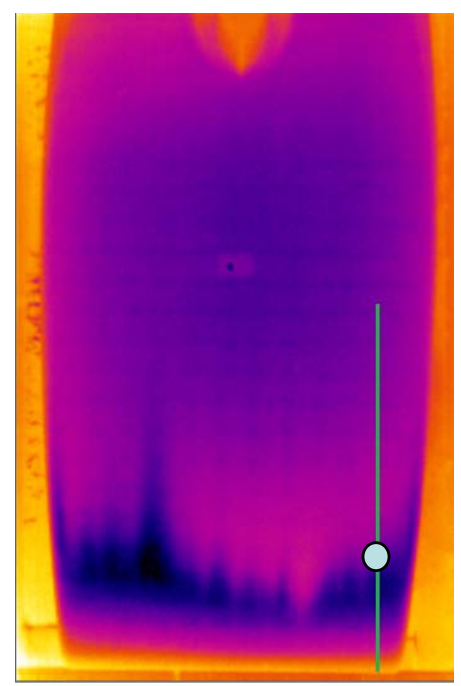

Figure $11-$ Test $n^{\circ} 2-U=40 \mathrm{~m} / \mathrm{s}$

The distance from the leading edge at which the transition occurs measured using the IR results has been compared with the theoretical position for a flat plate assuming a critical Reynolds number of 500.000. The results are given in Table 1. The positions obtained experimentally correspond to the transition position measured along the same line for the different tests.

Table 1 - comparison between the theoretical and measured position of transition

\begin{tabular}{|c|c|c|c|c|}
\hline $\mathrm{U}-\mathrm{m} / \mathrm{s}$ & 28 & 32.5 & 40 & 45 \\
\hline Theoretical position from leading edge - m & 0.27 & 0.23 & 0.19 & 0.17 \\
\hline Position detected with IR camera - m & 0.3 & 0.2 & 0.13 & 0.1 \\
\hline
\end{tabular}

The previous figures have shown that the position of the transition varies along the width of the model. To check if this phenomenon was due to differences in the material properties inside the model or results from the flow field above the model, the IR images have been compared with oil visualization. The results are shown in Figure 12 and in Figure 13 . The 
oil pattern observed in the first figure is qualitatively close to the shape of the transition region observed in the second figure. We can therefore conclude that the variation of the transition position along the width of the model is not related to the material properties of the model, but to a variation of the flow field above the model.

This result indicates that the model was maybe not perfectly oriented inside the flow field and that therefore transition occurs at different distances from the leading edge along the width. New tests will be performed with a more accurate control of the angle of attack and of the position of the model.

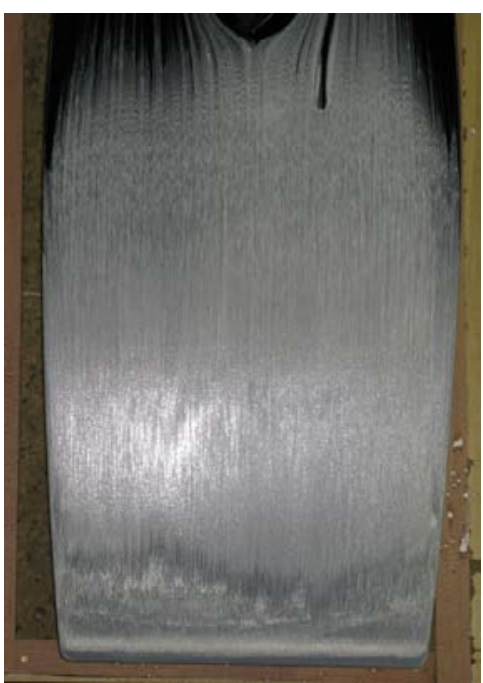

Figure 12 - Oil visualisation

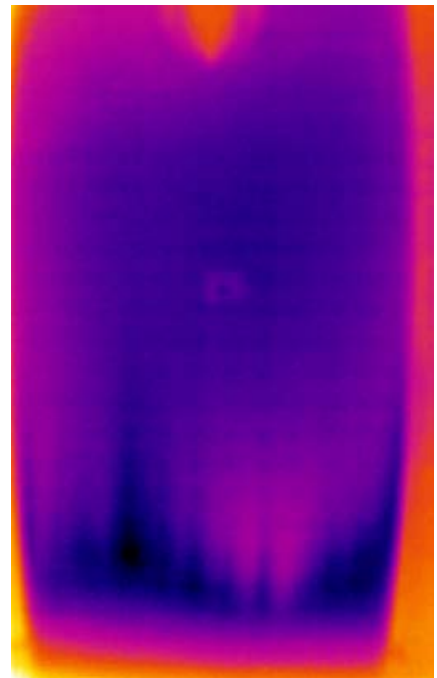

Figure 13 - Temperature distribution

\section{Conclusions and future work}

A new methodology for the transition detection, based on a initial cooling of the model and on the measurement of the time-dependent surface temperature evolution has been studied and presented in this paper.

The methodology is based on the use of the infrared thermography for the detection of the transition position on a scaled model of an Ultra-Streamlined body in a subsonic flow. The quantitative measurement of the heat transfer coefficient based on the infrared images and the use of a simplified heat transfer model has been described.

The technique needs to be improved by controlling in a better way the initial wall temperature but the technique can already be used for a parametric study.

Results on two different scale models of an ultra-streamlined body have been presented. Significant differences have been observed on the transition pattern on the upper part of the models. The tests on the second model have also shown a great dependency on the angle of attack of the model on the transition position.

\section{Acknowledgment}

The authors thank E. Labergère for his help during the present research, especially for the measurements using the second model shown in this paper.

\section{REFERENCES}

[1] Gauffre G. "Detection of laminar-turbulent transition by Infrared Camera," ONERA, La Recherche Aérospatiale, 1988.

[2] Bejan A., Heat transfer, John Wiley \& Sons Inc. (USA), 1993. 